\title{
An engineering-design oriented exploration of human excellence in throwing
}

\author{
SUSHEEL DHARMADHIKARI $^{1, *}$ and ANINDYA CHATTERJEE ${ }^{2}$ \\ ${ }^{1}$ Structural Dynamics Team, Eaton Technologies, EON IT Park, Pune 411014, India \\ ${ }^{2}$ Department of Mechanical Engineering, Indian Institute of Technology, Kanpur 208016, India \\ e-mail: sushildharmadhikari7@gmail.com; anindya100@gmail.com
}

MS received 3 November 2016; revised 12 March 2017; accepted 19 July 2017; published online 10 March 2018

\begin{abstract}
Humans are fast throwers, and their bodies differ correspondingly from those of other hominids. One might ask why humans evolved to throw fast while others did not; whether the design of a fast thrower is unique or special and whether indeed humans remain fast within broader comparison sets of non-hominid throwers. As a non-hominid comparison set, we consider a random population of five-link robots with simplified joint angle and torque constraints. We generate 20,000 such robot models and sequentially optimize their throwing motion. Since good initial guesses are needed for each optimization, the robots are first arranged in distance-minimizing sequences in design parameter space. Each robot's optimal throw then serves as an initial guess for the next one in sequence. Multiple traversals of these sequences, and random perturbations, are used to avoid local optima. Subsequently, regression models are used to predict throwing performance as a function of robot design parameters. From these regression models, the dominant heuristic predictor of fast throwing is found to be a long and light last link. Direct optimization of the robot design leads to much faster throwers, also with long and light last links. In striking contrast, the human arm has two equally long intermediate links of significant mass. Nevertheless, a somewhat human-like arm within the same robot set is found to be a good thrower. On combining several throwing criteria to obtain a single figure of merit, the human-like arm lies in the 96th percentile of the population. Since our human-like arm is a crude approximation of an actual human arm, we suggest that fast throwing by human-like robot arms is not inherently difficult from a mechanical point of view.
\end{abstract}

Keywords. Human arm; throwing; robots; optimization; statistics.

\section{Introduction}

Humans excel at fast throwing. They throw small projectiles faster than any other animal on Earth. Consequently, the biomechanics of human throwing has been well studied in the research literature. Yet, human throwing is a selflimiting subject. It does not ask how other animals might or might not learn to throw fast, or whether evolving to throw fast is somehow inherently difficult, and so on.

Interestingly, hominids have been around for about 80 million years, while Homo habilis appeared only about 3 million years ago. In other words, roughly human-like arms have been around for a long time, while fast throwing prowess has developed only recently.

As engineers taking a design viewpoint, we wonder why fast throwing took so long to develop. Was the evolutionary process slow and inefficient? Is a fast-throwing animal design particularly difficult to find? Or, was it simply not beneficial to throw fast without accompanying development

*For correspondence of trajectory estimation (read: throwing aim)? Consider a hypothetical researcher from the pre-human period, e.g., 5 or 15 or 60 million years ago, studying throwing in primates. Such a researcher might have incorrectly concluded that primates cannot be good throwers. Musing further, might another game of the evolutionary dice have resulted in, say, slender flexible elephant-like trunks that were fast throwers? These speculations are meant merely to indicate that a study of strictly human throwing is, in a way, circular and self-limiting.

Note that discussion of optimality in specifically-human throwing is difficult because human arms do many things, e.g., load lifting, climbing, balancing, feeding, fighting, gripping and, generally speaking, both static and dynamic manipulation of small and large objects. Hence, even if a human arm is optimized for throwing, that optimization is necessarily subject to complex multiple constraints that we may not be able to accurately formulate.

With these musings, as mechanical engineers looking at a throwing animal from a design viewpoint, we suggest here that some useful questions can be asked that are easier 
to answer, and which may shed some indirect light on human throwing. To that end, we approach the excellence of human throwing motion from a statistical and mechanistic perspective rather than a human-centric and biomechanical one.

Our intention here, we emphasize, is not to undermine the human-centric and biomechanical approach, but rather to complement it. We note here the detailed and authoritative treatment of [1], which strongly adopts the human-centric and biomechanical view. For a discussion of gripping from an evolutionary viewpoint, see [2]. Wood et al [3] discuss how some perceptual abilities that are required by throwing preceded the evolution of the motor skills relevant to throwing. Hopkins et al [4] studied neurological adaptations related to throwing and noted differences in brains of chimpanzees that correlated with variations in their throwing ability. It is thus clear that anatomy and biomechanics play critical roles in human throwing, and the ability to throw fast has evolved over time. There are also various studies that address throwing biomechanics from a injury avoidance viewpoint, e.g., [5, 6] and [7].

In contrast with the above hominid-centric studies, we have found no research on the superiority of human throwing within a non-hominid comparison set. In this paper, we study throwing in a large random population of robotic arms in a mechanical design setting. Our study of a human-like arm within this set of robots is almost incidental. Biological aspects like tendon elasticity, joint compliance and injury are deemphasized. By our mechanistic focus on a large set of simple robots and their optimized throwing motion, we deliberately move the discussion away from humanspecific biomechanics and into more routine rigid body mechanics, hoping for insights into throwing by both robots and humans.

Our robots have simple geometry, a small number of degrees of freedom (five rigid links), simplified torque limits and joint angle constraints that do not favour humans and no weight (i.e., we neglect gravity), and a small pointmass projectile is considered. We will study the individually optimized throwing motion of 20,000 such robots with randomly generated physical parameter values. Our intention here is twofold: to see how robot parameters may predict achievable throwing speed and see also how a somewhat anthropomorphic arm measures up against this population of random robots.

Our main technical finding is that the anthropomorphic arm does unexpectedly well against the random population: "unexpectedly" in the sense that the arm does better than a regression model based on robot parameters would suggest. A less obvious, but also interesting, point is that the anthropomorphic arm is at best a simplified and restricted version of a human arm; and hence its good throwing performance suggests that good human-like throwers are in some sense not too difficult to design. In this way, our work is complementary to the human-centric studies mentioned earlier.

Before proceeding with the technical content of the paper, we mention here for completeness some relevant works on throwing motion of robots. Lombai and Szederkényi [8], Kato et al [9], Sato et al [10] and Senoo et al [11] focus on algorithms for dynamic motion planning and control for given robots. It is also, of course, possible to model human-like throwing motion in detail [12]. Finally, there is significant research on the biomechanics of human throwing (see e.g., [13-15] and [16]).

All in all, we have seen no paper that assesses humanlike throwing using a mechanistic comparison set as we do in this paper.

\section{Robot population details}

We first describe the robot model. Kinematics calculations referred to here are described in detail in the appendix.

\subsection{Model}

A human thrower uses many joints as well as flexibility. For simplicity, we consider only 5-link rigid spatial robots with revolute joints. Figure 1 shows a sketch of such a robot. In the figure, the $\hat{n}$ s are unit vectors along joint axes and the $\hat{x}$ s are unit vectors along the links.

Some anthropomorphic arm geometries are included within such 5-link robots; for example, two short links with mutually perpendicular joint axes can mimic the shoulder, with the next two longer links mimicking the upper and lower arms, and a short fifth link mimicking the hand. Note also that the joint axis directions are design parameters that change from robot to robot, and so a large range of motion is possible within the set of robots being considered.

The robot model is idealized and simplified as follows. All links are treated as straight, thin, uniform rigid rods. Selfcollisions of links are ignored. Gravity is assumed to be negligible. Equal and opposite torques act implicitly at each revolute joint as needed, with motor masses and other characteristics ignored. The projectile to be thrown is a point mass attached to the tip of the outermost link. By appropriate choice of units, the total mass of the five links (not including the projectile) is taken to be unity, and the projectile mass is arbitrarily taken to be 0.1 (small, but not negligible). Similarly, the sum of the five link lengths is taken to be unity. All five joint rotation angles $\xi_{i}(i=1,2, \ldots, 5)$ are assumed to be restricted within some range, i.e., $\xi_{i, \max }-\xi_{i, \min } \leq \Phi$ for some $\Phi$. We have studied two cases: $\Phi=\pi$ and $\Phi=\pi / 2$. Finally, the sum of the absolute values of the five joint torques is taken to be bounded by unity at all instants during the throwing motion (an admittedly arbitrary choice: we will explain our reasons later). 


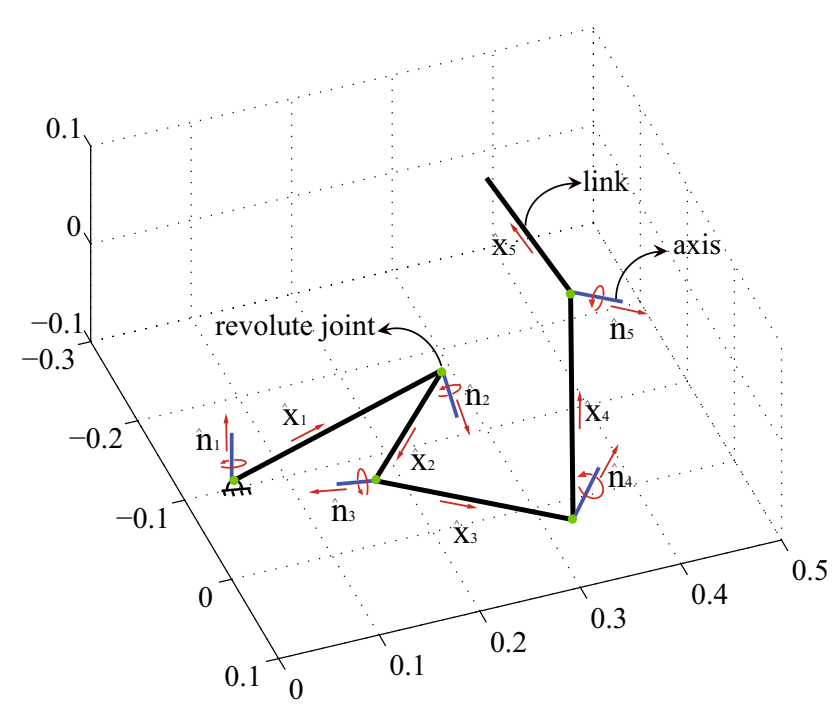

Figure 1. Five-link robot. The first link is pivoted at the ground. The blue line segments denote successive joint axes, fixed to successive links: $\hat{n}_{1}$ is fixed relative to ground, $\hat{n}_{2}$ is fixed relative to link 1 , etc.

\subsection{Parameters}

The physical parameters that define a robot can now be enumerated.

Without loss of generality, since gravity is ignored, the first links' pivot axis with ground is taken to be along the vertical or $z$-direction. The remaining four joint axes in the robot's reference configuration can be specified using two angles each, giving eight parameters.

Unit vectors along each of the five links can similarly be specified using two angles each, giving 10 parameters. Since the lengths add up to unity, only four lengths need to be specified. Similarly, only four masses need to be specified. Thus, there is a total of 26 parameters.

A given robot could be shifted to a different reference position through five arbitrary joint rotations, giving a seemingly different set of 26 parameters. To make the reference position unique and also enable numerical comparison between different robot designs, a simple optimization problem is solved for each robot: the tip of its outermost (fifth) link is moved as far out in the $x$-direction as possible. For ease of reference below, this optimization will be referred to as " $x_{\text {tip }}$-maximization". After this maximization, technically there remain only 21 independent parameters that specify a robot. However, eliminating parameters seemed awkward, and we retained all 26 parameters at the values obtained after carrying out the maximization.

Thus a robot design, $D$, is a column matrix containing 26 numbers (after $x_{\text {tip }}$-maximization). The designs were randomly generated as described later.

\subsection{Population}

First, consider generating a single robot design $D$. Four unit vectors along joint axes and five unit vectors along links need to be generated (nine in all). For each such random unit vector, all directions in 3D space were taken as equally likely. Accordingly, for each vector, first a $3 \times 1$ vector of standard normal variables was generated (using the Matlab command "randn(3,1)"). Subsequently, that vector was normalized to unit magnitude, and then the unit vector was reduced to 2 angles (latitude and longitude, or azimuth and elevation). For link masses, 5 numbers uniformly distributed in the interval $[0,1]$ were first selected using the Matlab command "rand $(5,1)$ ". Subsequently, these numbers were divided by their sum, to make the sum unity, and the first 4 were retained as independent parameters. Link lengths were generated similarly. Finally, $x_{\text {tip }}$-maximization was carried out, as explained earlier. Matlab's "fminsearch" was used, and worked quickly.

We turn next to generating a population of 20,000 robot designs, geared towards subsequent throwing optimization calculations with them.

With our available computational resources, we found it convenient to run 16 simultaneous optimization loops on several desktop PCs running Matlab. With the constraint of 16 in mind, we first generated 4000 random designs as described earlier (with $x_{\text {tip }}$-maximization). These 4000 designs were partitioned into 16 subsets of 250 each, by repeatedly dividing into two $\left(16=2^{4}\right)$, using the algorithm ${ }^{1}$ of [17]. The intention was that each subset contains members that are close together in parameter space. Our next goal, within each subset, was to arrange them in a sequence such that the average distance between successive designs was small. To this end, we used the travelling salesman problem (TSP) solver tsp_ga from Matlab Central. $^{2}$ With the TSP solutions each subset had 250 designs arranged in a loop, because the travelling salesman returns to his starting point. Finally, by linear interpolation, 4 designs were inserted between every successive pair in the loop, taking the subset size (and loop length) to 1250 . The advantages of using the TSP in this way are that, in subsequent sequential optimization of throwing motion (to be described later), (i) each design's optimum throw can be used as the initial guess for the next design in the loop, (ii) the loop can be traversed in both directions and (iii) later on, suspiciously poor optima can be perturbed randomly or otherwise improved, and then new searches with these new initial conditions can be started in both directions from such points. In particular, step (iii) was repeated until it became difficult to find improvements and the extra effort did not

\footnotetext{
${ }^{1}$ Matlab code was obtained from http://homepages.cae.wisc.edu/ $\sim$ ece556/matlab/kldemo.m (we slightly modified the input and output).

${ }^{2}$ http://in.mathworks.com/matlabcentral/fileexchange/13680-travel ing-salesman-problem-genetic-algorithm/content/tsp_ga.m, by Joseph Kirk.
} 


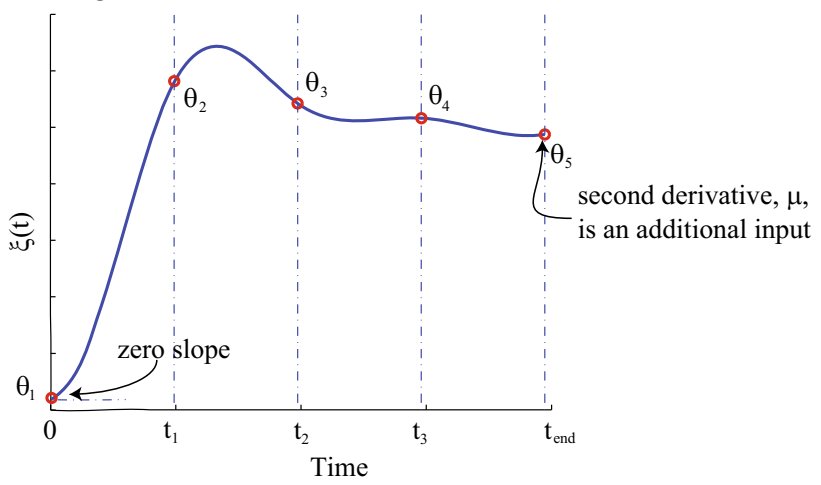

Figure 2. Joint-angle time histories are specified using cubic splines.

seem worthwhile. Each such set of throwing optimizations, over all 16 loops, with all PCs running almost constantly, took slightly above 3 weeks. For $\Phi=\pi$ and $\Phi=\pi / 2$, this is about 7 weeks. Including initial time spent in developing the strategy and automating the computations, the total computational time spent was about 4 months. Later researchers with clearer strategies and faster computers may require less time.

\section{Constrained fast throwing}

We now formulate, and computationally solve, the throw optimization problem.

\subsection{Notation}

3.1a Time history of joint angles: We specify joint angle histories as a function of time $t \in\left[0, t_{\text {end }}\right]$ using cubic splines. Values of the angle $\xi$ are specified at five uniformly spaced points in time. Two additional conditions are needed, and they are chosen to be a zero derivative at $t=0$ (starting from rest), and a specified second derivative $\mu$ at $t=t_{\text {end }}$ (implicitly influencing joint torques at the instant preceding release). Figure 2 shows a schematic diagram of the same. For each such time history, there are 6 input parameters ( 5 nodal values and 1 final second derivative $\mu$ ). For 5 joint angles' time histories, there are thus 31 input parameters, including $t_{\text {end }}$. Thus, a throw is a 31-dimensional vector, which we denote by $\Psi$.

3.1b Angle constraints: It is in principle possible to generate high throwing speeds using finite torques by spinning the robot many times round and round, accelerating all the while. To remove such motion from consideration, we force $\xi_{i}(t), i=1,2, \ldots, 5$ (the 5 joint angles), to be restricted within a specified range, i.e.,

$$
\max _{t \in\left[0, t_{\text {end }}\right]} \xi_{i}(t)-\min _{t \in\left[0, t_{\text {end }}\right]} \xi_{i}(t) \leq \Phi, \quad i=1,2, \ldots .5
$$

where $\Phi$ was taken to be $\pi$ and $\pi / 2$ in two different sets of optimizations. The maximum and minimum of each joint angle was not computed merely at the 5 nodal points used in the spline, but at 45 equally spaced points where each spline was evaluated, for higher accuracy.

Note that the average value of the joint rotation angle was not restricted in any way. The robot during throwing was not required to remain close to its reference position as obtained through $x_{\text {tip }}$-maximization as described earlier.

3.1c Torque constraints: Given all masses, lengths, orientations and joint angle histories, we use inverse dynamics to compute the implied torques (see the appendix). The torques need to be constrained in some way, to keep throwing speeds bounded. These torques are complicated functions of design parameters and joint angle spline data, and hence constraining their maximum magnitudes using closed form analytical expressions is impractical. We enforced torque limits using penalty weights instead.

Note that there are three fundamental physical dimensions in our problem, namely mass, length and time. We have already restricted the total mass and the total length to be unity, which is equivalent to eliminating two free parameters through non-dimensionalization. Similarly, limiting some suitable torque to unit magnitude is a way of indirectly non-dimensionalizing the system using time.

We encountered a philosophical dilemma in choosing the torque quantity that we would limit to unity. We first contemplated limiting each joint torque to unit magnitude. However, such uniform joint torque limits seem arbitrary if the links have variable lengths, masses and orientations. At the same time, not using uniform torque limits seemed arbitrary as well. For example, introducing more humanlike limits (e.g., larger torques near the shoulder and smaller torques near the wrist) might give an undue advantage to the human thrower, which is something we wish to avoid. Yet again, there are also practical difficulties: with separate inequalities for each joint torque, we found there is a greater tendency to get stuck in local minima, greatly increasing the time spent in searching for global, or near-global, optimal values.

As a practical choice, therefore, the torque limits were distributed equally over all joints, and we adopted a constraint on the sum of torque magnitudes, i.e.,

$$
\sum_{i=1}^{5}\left|\tau_{i}(t)\right| \leq 1, \quad t \in\left[0, t_{\mathrm{end}}\right]
$$

which was actually checked at 45 points as mentioned earlier. Note that Eq. (2) is not motivated by biomechanics and does not specifically favour human-like throwers. It is also simple, allows a large range of throwing motion and computationally requires just a single penalty weight. 


\subsection{Optimization problem and numerical solution}

Our optimization problem was as follows:

$\max _{\Psi}$ kinetic energy of the tip mass,
subject to inequalities $(1)$ and $(2)$.

We used a combination of penalty terms and Matlab's fmincon for the optimization. The angle and torque constraints were enforced using a penalty parameter. We also imposed bounds on the final angular acceleration $\mu$. Bounds on $\mu$, and other bounds we used on $t_{\text {end }}$, were imposed using fmincon. These bounds were used to aid the search but were irrelevant for the final optima obtained. In all solutions finally presented here, the constraints on $\mu$ and $t_{\text {end }}$ were inactive.

Note that the optimization problem is non-convex, and must be solved individually for 20,000 robots. Many random initial guesses need to be tried, and knowledge of optima for "nearby" robots needs to be used, to obtain an overall reliable set of 20,000 optimal or near-optimal solutions. The complete task was large, and done as follows.

The optimization problem was solved in 16 parallel loops over as many subsets of the robot population, as described earlier. For each loop, we first selected a design at random, chose many initial guesses for it, and found a good optimum for it. Then the loop was traversed sequentially, with the previously found optimal throw used as an initial guess for the next design.

All loops were traversed more than once; many designs were reexamined by perturbing their optimal throws and optimizing again; points where significant improvements were obtained were used to start new local searches for improvements at nearby designs on the same loop; points where the throw was suspiciously poorer than those at neighbouring points were perturbed and reexamined, and in this way, through multiple and persistent searches, good optima were found for almost every design.

We stopped when new improvements became unreasonably difficult to find, and after multiple loop traversals. A fraction of the population may remain trapped in local optima, as is unavoidable for such a large number of nonconvex optimization problems. ${ }^{3}$ As mentioned earlier, this set of optimization problems represent about 7 weeks' worth of multiple desktop PCs running round the clock. Although there are no formal guarantees, we do believe that these results cannot be significantly improved without a paradigm change in computational resources. We therefore accept these results, and move on to a statistical assessment of the population's throwing motion.

\footnotetext{
${ }^{3}$ Remaining trapped in local optima for biomechanical tasks is easy even for intelligent humans. Consider the many talented high jumpers who never thought of the justly famous Fosbury Flop.
}

\section{Initial results and correlations}

We found optimal throws for all 20,000 robots and for two values of $\Phi$, namely $\pi$ and $\frac{\pi}{2}$. Histograms for these two sets of throws are shown in figure 3 .

We now consider statistical correlations. The maximum constrained-throwing kinetic energy (KE) achievable by a given robot is a function of its 26 design parameters. ${ }^{4}$ Thus, we sought regression models that fit the optimized kinetic energies as simple functions of robot parameters.

To this end, we found that the correlation between actual $\mathrm{KE}$ and the predicted (or fitted) $\mathrm{KE}$ from a linear regression model was 0.72 for the $\Phi=\pi$ throws, and 0.80 for the $\Phi=\pi / 2$ throws. In these calculations, for each dataset of 20,000 optimized throws, we actually used two random subsets of 10,000 each, one for fitting and one for subsequent testing, and results across these subsets were consistent (with correlations for the test data being very slightly inferior).

Note that a linear regression model in this case has 27 fitted coefficients (including an added constant). The correlations improved with a quadratic model with all possible square and quadratic terms included, which has 378 fitted coefficients in this case. For $\Phi=\pi$, the correlation improved to 0.75 , and for $\Phi=\pi / 2$ to 0.83 ; once again the test data correlations were only slightly inferior.

Finally, for a third-order model (3654 coefficients), the correlations for the fitting data improved while those for the test data deteriorated, and hence those results were not acceptable. In separate attempts to fit the data using neural networks from Matlab's toolbox, we obtained results comparable to those of the quadratic model; those attempts are not documented here.

Our unexpected conclusion from the regression models is that the maximized KE apparently cannot be fitted as a simple function of design parameters. Note that the square of our reported correlation is the more popular " $R^{2}$ " of statistical regression, and the best $R^{2}$ we achieved was under 0.7 .

In search of simpler descriptions, we noted the remarkable paper of [18]. Dawes observes that in situations where predictions are poor (i.e., $R^{2}$ is low), relatively good models can be obtained by retaining a few key variables, normalizing them to unit variance, and assigning them weights of \pm 1 , the sign being chosen to reflect the direction in which each independent variable affects the fitted variable. Considering our 26 design variables, we found that two of them had relatively strong correlations with the optimized $\mathrm{KE}$ : the last link's length (positive correlation) and mass (negative correlation). Accordingly, we considered the correlation of the maximized $\mathrm{KE}$ with the simple predictor $L_{5}-M_{5}$ (fifth length minus fifth mass). The results were

\footnotetext{
${ }^{4}$ Due to $x_{\text {tip }}$-maximization 5 of these parameters are redundant, but not in a way we could easily exploit.
} 

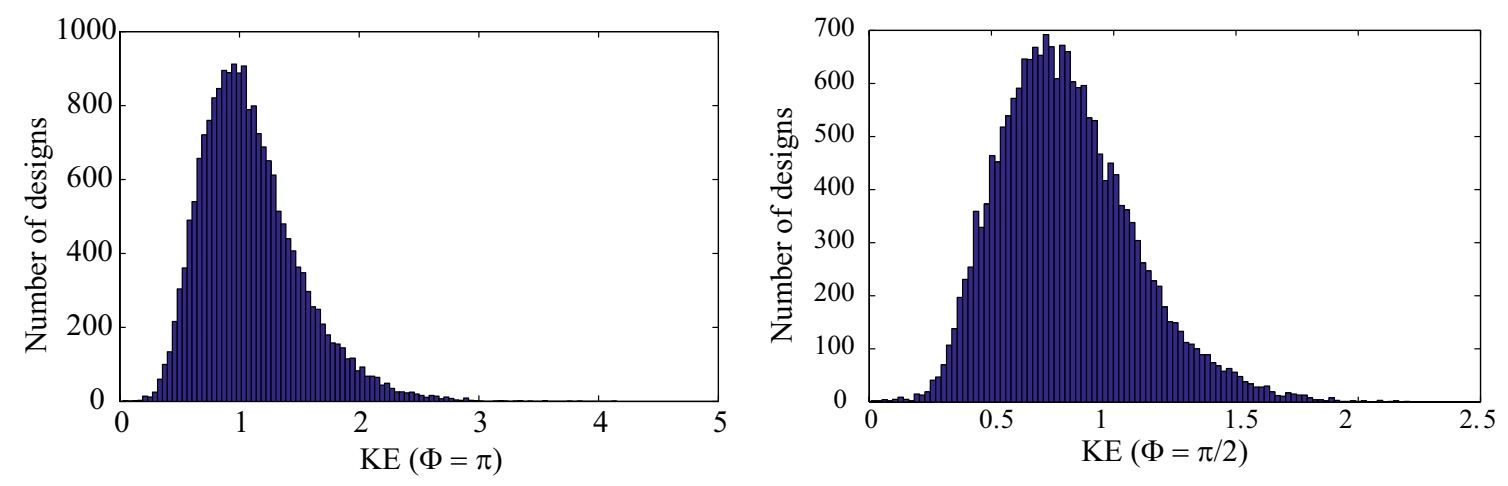

Figure 3. KE histograms. Left: $\Phi=\pi$. Right: $\Phi=\pi / 2$.
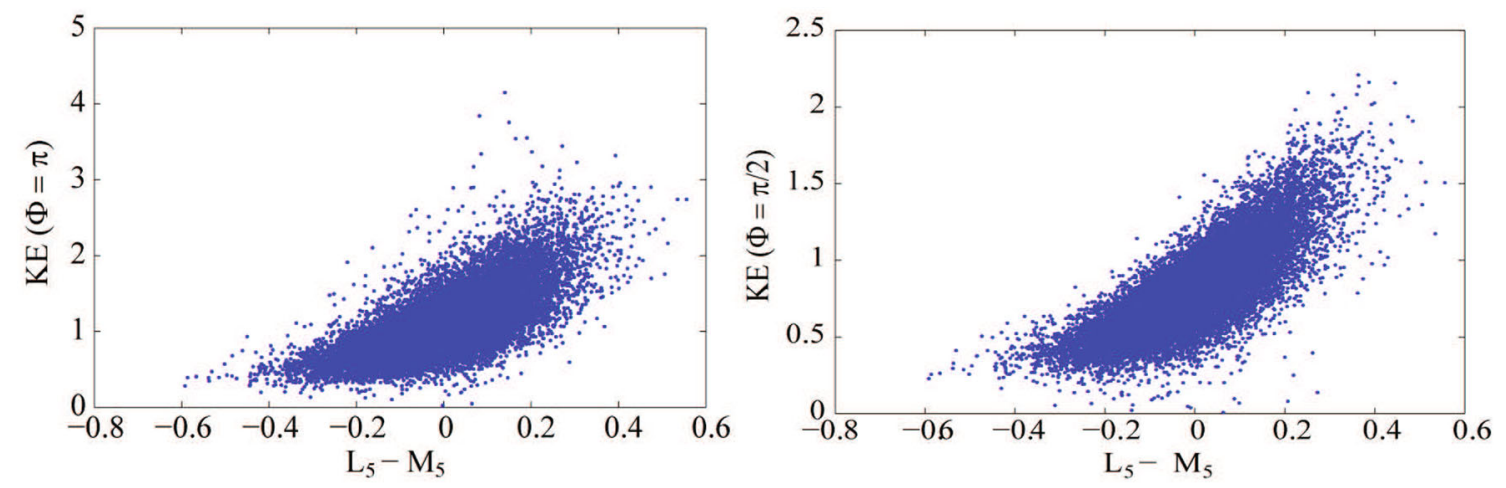

Figure 4. Scatter plot of $K E$ vs $L_{5}-M_{5}$. Left: $\Phi=\pi$. Right: $\Phi=\pi / 2$.

surprisingly good: 0.71 for $\Phi=\pi$ and 0.79 for $\Phi=\pi / 2$. These correlations were the same for both fitting and test data.

The scatter plot of the KE of the tip mass vs $L_{5}-M_{5}$ is shown in figure 4.

The net result of this preliminary search for good correlations is that a long and light last link seems to favour fast throwing. By this criterion, an anthropomorphic robot arm seems to be at a disadvantage. However, the correlation is far from perfect, and there is room for human-like arms to throw well regardless of figure 4 .

\section{Three new robots}

We now have a background set of non-human-centric throwers. With them available to provide context, we finally study three new robots.

The first two are robots that are themselves optimized to be excellent throwers, one for $\Phi=\pi$ and one for $\Phi=\pi / 2$, both within the mechanical assumptions of our robot population ( 5 slender uniform rigid links, 5 revolute joints, sum of masses equals unity, sum of lengths equals unity). Thus, the 26-dimensional design parameter vector and the 31-dimensional throw-parameterization vector are combined to form a 57-dimensional vector, which is optimized to obtain the fastest possible thrower we can find.

Figure 5 shows the fastest thrower found for $\Phi=\pi$. The throw (indicated by red) is significantly better than the population, and consistent with a large value of $L_{5}-M_{5}$, as suggested by foregoing results. Figure 6 shows a sketch of this robot. Its $L_{5}=0.7176$ and $M_{5}=0.0010$.

Corresponding results for the $\frac{\pi}{2}$ case are shown in figures 7 and 8 . In this case, we found $L_{5}=0.5497$ and $M_{5}=0.0021$.

These two optimized thrower designs may not be suitable for many animals. For example, an extremely long and light last link might lack strength and stiffness as well as fail at some tasks like bringing food to the mouth. With these thoughts, we turn to the third and last robot arm considered in this paper. This is a roughly anthropomorphic $\mathrm{arm}^{5}$ as shown in figure 9. The proportions of the masses are taken from [19]. ${ }^{6}$ Length proportions used are based on our own rough estimates. There is some arbitrariness in the choices of the joint axes indicated in the figure. We have

\footnotetext{
${ }^{5}$ The arm can be fully straightened; lengths and joint axes are as sketched and the link masses, from shoulder to hand, are taken as 0.02, $0.02,0.53,0.38$ and 0.05 . Slightly better throws are obtained if we shift some of the mass to the first 2 short links, but we avoided that trick.
}

${ }^{6}$ Our source: http://www.exrx.net/Kinesiology/Segments.html. 

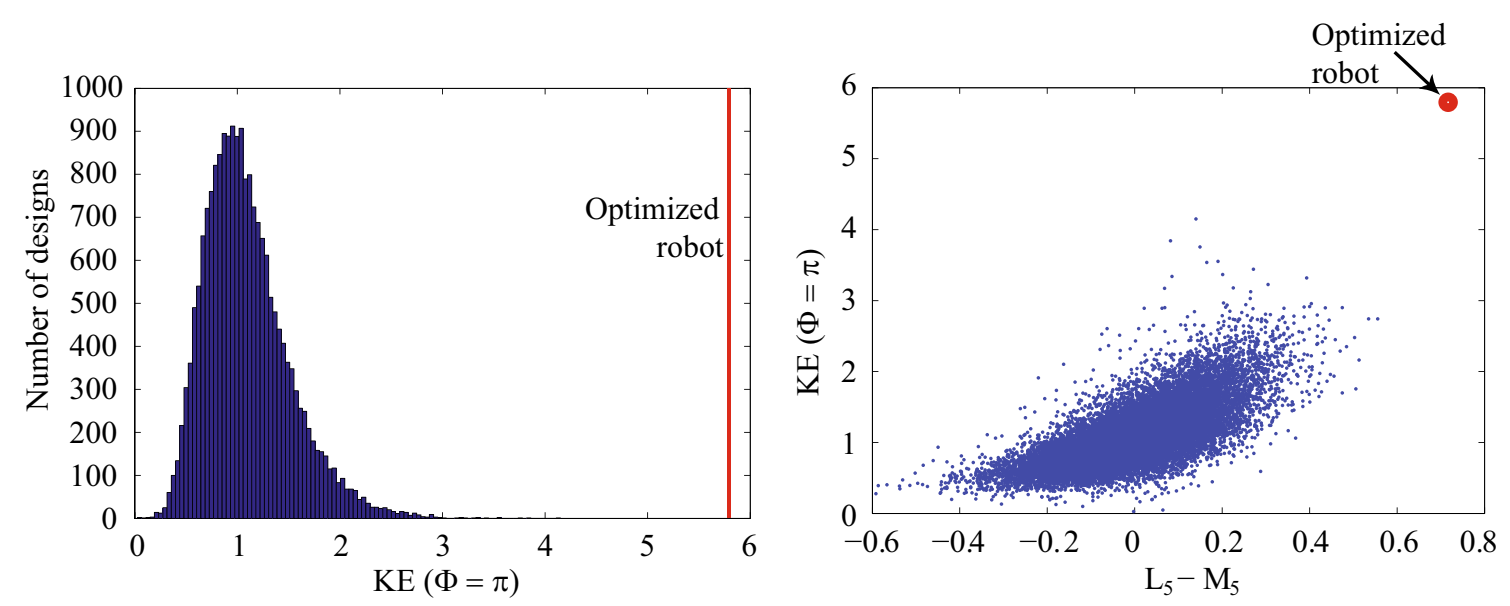

Figure 5. Fastest-throwing robot found for $\Phi=\pi$. Left: histogram. Right: scatter plot.

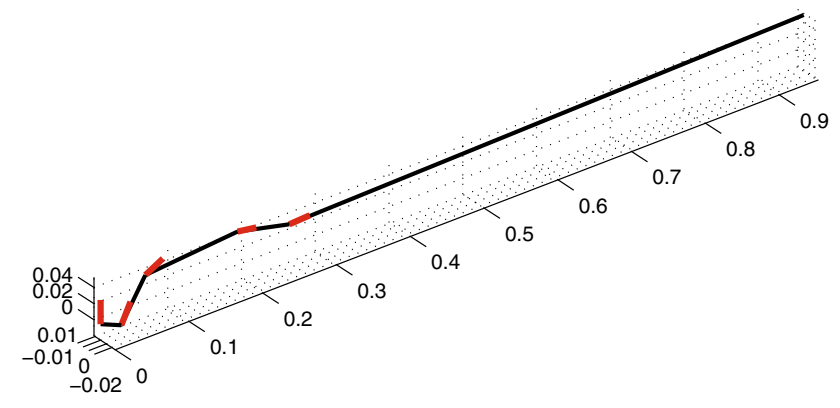

Figure 6. Optimized robot design $(\Phi=\pi)$. The red line segments denote successive joint axes, fixed to successive links.

sought optimal throws for this robot arm for both $\Phi=\pi$ and $\Phi=\pi / 2$. Sketches of the optimized throwing motion are given in figure 10. There is a superficial resemblance to overarm bowling in cricket, but the elbow bends significantly.

The comparison with the robot population is shown in figures 11 and 12. Although it cannot match the two optimized throwers, the human-like arm has a relatively high rank with respect to the overall population $\left(98^{\text {th }}\right.$ and $92^{\text {nd }}$ percentiles). We examine this further later.

\section{Additional criteria for throwing}

We have, for all robot designs, optimized the throws for maximum speed of the thrown mass. However, these optimized throws can be evaluated using additional criteria as well. Two such criteria follow.

The first criterion we consider is simply the energy efficiency of the throw, i.e., the ratio of the KE of the thrown point mass to the total KE at the instant of release.

For our second criterion, we note that one reason why humans throw objects is to try and hit targets. We suggest that the dominant part of the throwing motion is geared towards generating speed in roughly the right direction, while the last few moments of throwing may also be relevant for perfecting one's aim. One aspect of the throw that is easy to adjust, very late in the throw, is the instant of release: in comparison, other motion quantities are difficult to change very late in the throw. If the instant of release is slightly perturbed, the acceleration at the instant of release determines the resulting change in the velocity of the throw. If this acceleration vector is along the velocity of release, then only the throwing speed is affected. However, if the acceleration is perpendicular to the velocity of release, then the robot tip motion preceding release is along an arc, and the throwing direction is affected. The idea is illustrated in figure 13. In the figure, the two dashed arrows describe a triangular region, and this region may be thought of as the region accessible to the thrower.

With these thoughts, we suggest a final criterion for evaluating throws: a high value of $\left|\vec{a}_{\text {tip }} \times \vec{v}_{\text {tip }}\right|$. We denote this quantity, which is proportional to the area of the triangular region mentioned before, as

$$
A=\left|\vec{a}_{\text {tip }} \times \vec{v}_{\text {tip }}\right| .
$$

\section{A single figure of merit}

As observed in section 5, the two robots optimized for throwing with $\Phi=\pi$ and $\Phi=\pi / 2$ are both excellent throwers. However, they have excessively long and light last links, and may be biomechanically unsuitable. We consider them no further.

The anthropomorphic arm has, somewhat surprisingly in light of the dominant statistical correlation before, been found to be a fairly good thrower. ${ }^{7}$ Here, we try to assess its

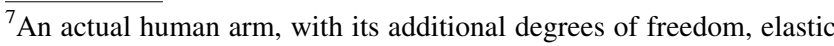
storage, etc., may be even better.
} 

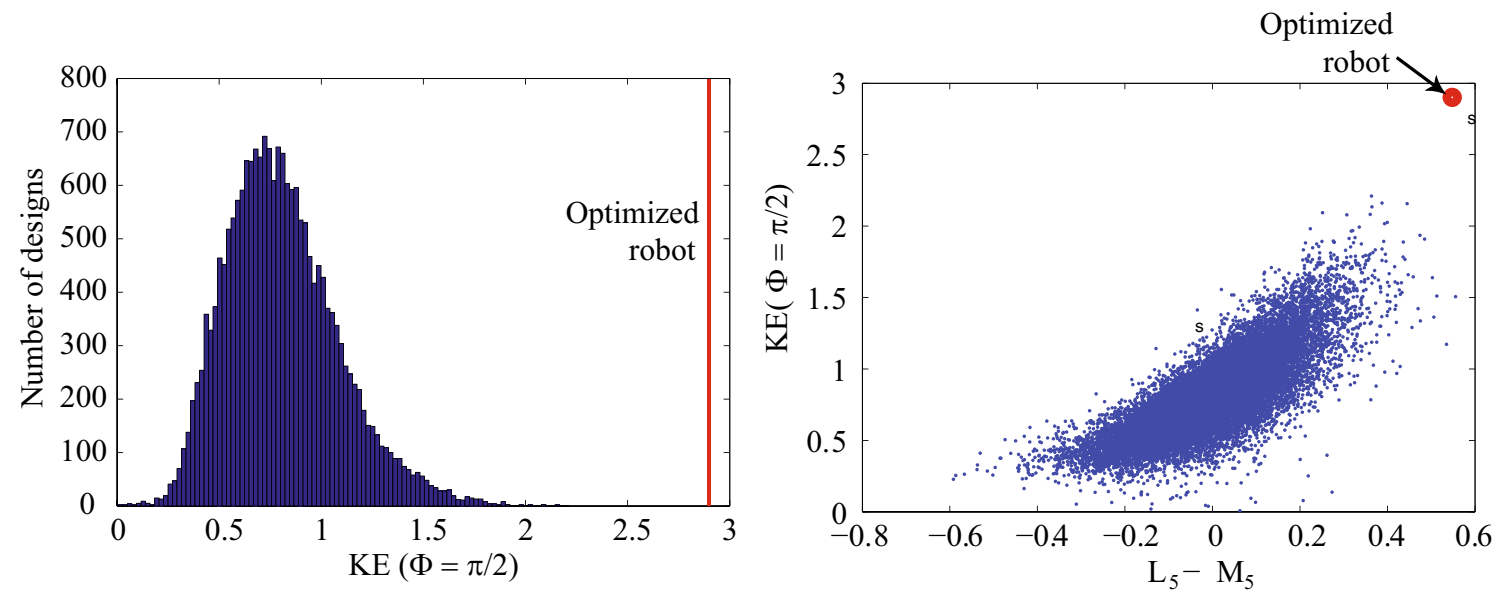

Figure 7. Fastest-throwing robot found for $\Phi=\pi / 2$. Left: histogram. Right: scatter plot.

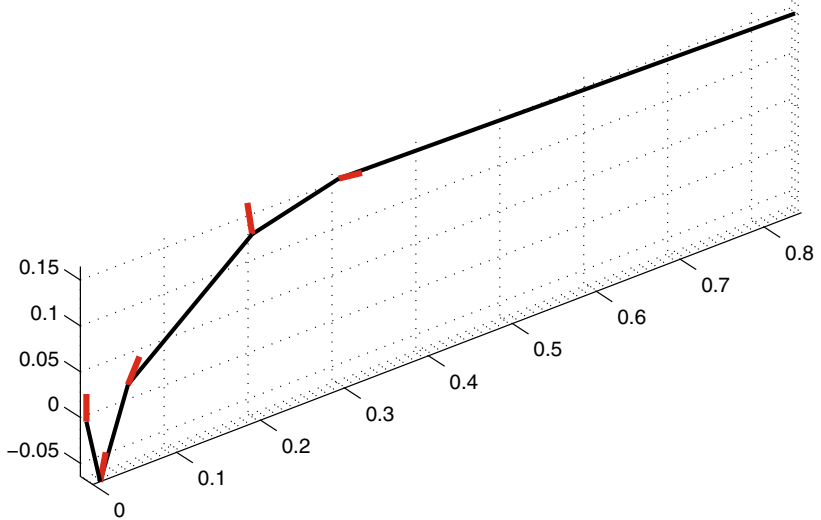

Figure 8. Optimized robot design $(\Phi=\pi / 2)$. Red line segments denote joint axes on successive links.

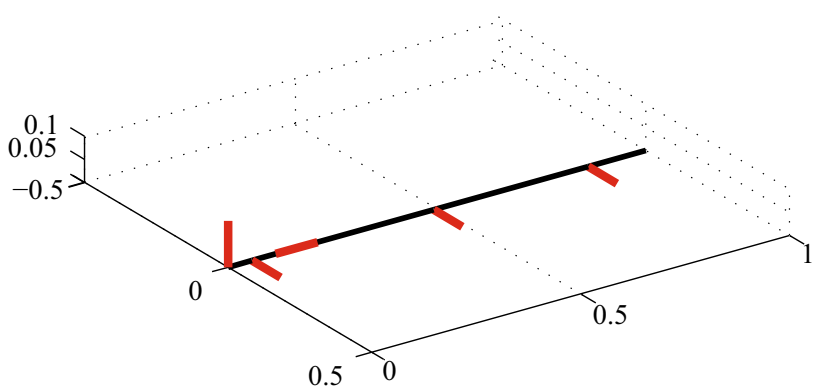

Figure 9. An anthropomorphic robot arm. Two short links mimic the shoulder, the next two mimic the upper arm and forearm (of roughly equal length) and the last link mimics the hand. Red line segments denote joint axes.

throwing ability using six different criteria as follows: for each of $\Phi=\pi$ and $\Phi=\pi / 2$, we consider the $\mathrm{KE}$ and energy efficiency $(\eta)$ as well as the $A$ parameter of Eq. (3). Since these 6 quantities are physically different, we assess the performance of the human-like arm in terms of its

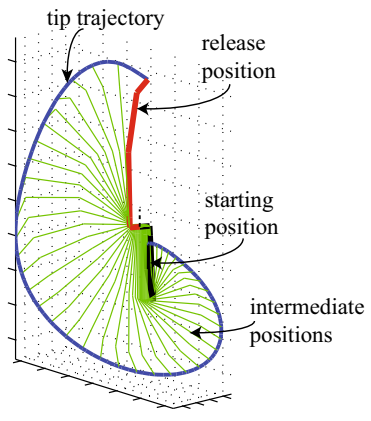

(a) $\Phi=\pi$

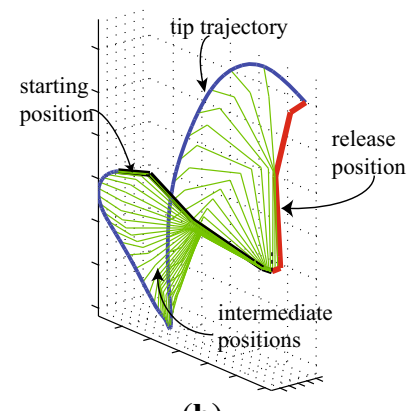

(b) $\Phi=\pi / 2$
Figure 10. Optimized throws of the human-like arm.

percentile score on these criteria, relative to the random population of 20,000 robots. These percentile scores are found to be the following.

\section{For $\phi=\pi$ :}

KE: $97.75, \eta: 90.26, A: 95.4$.

2. For $\Phi=\pi / 2$ :

KE: $91.94, \eta: 88.98, A: 87.02$.

It remains to combine these 6 measures of throwing performance into one reasonable figure of merit for the humanlike arm. To this end, we have simply added up the 6 percentile scores for the human-like arm, as also the corresponding 6 scores for all 20,000 robots. Taking this total score as an ad hoc figure of merit, the human-like arm is found to have a percentile score (relative to the population) of 95.98 .

This high final percentile score is, to us, the main finding of this paper. We summarize it as follows. Statistical correlations suggest, and optimized throwers demonstrate, that rather non-human-like arms might be the best throwers. Nevertheless, a human-like arm, not obtained from 

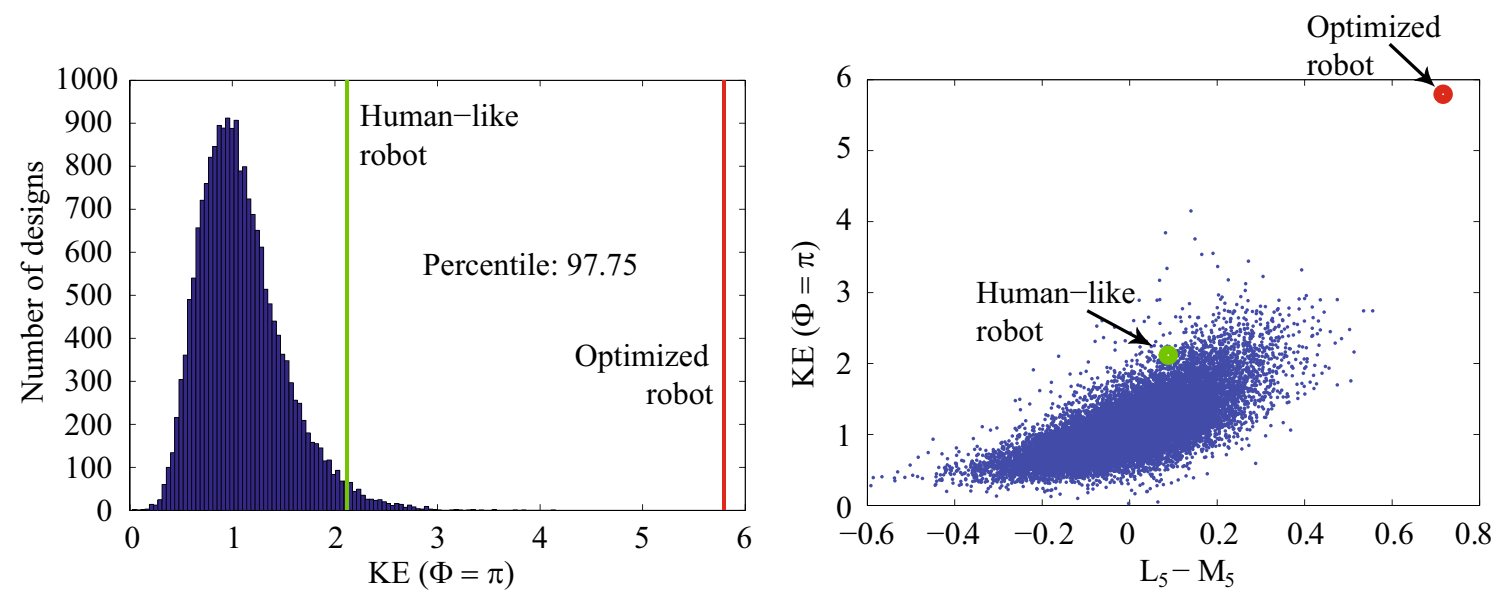

Figure 11. Human-like arm, $\Phi=\pi$, histogram and scatter plot.
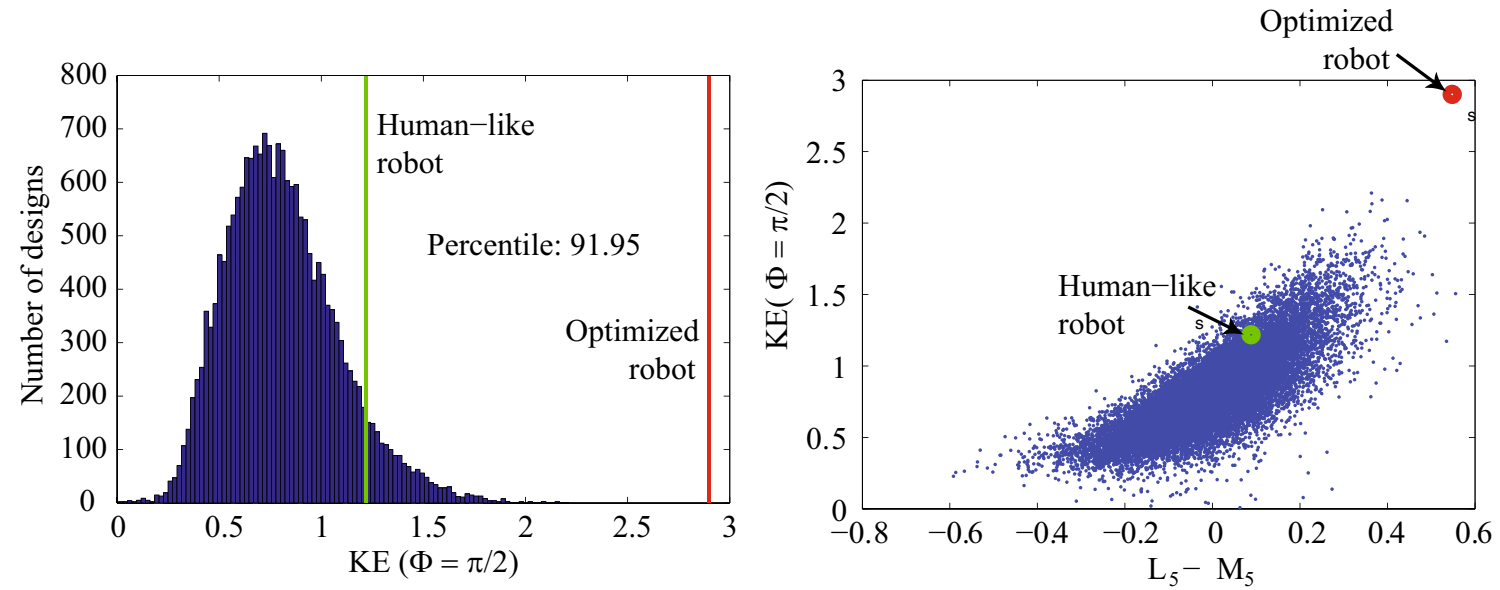

Figure 12. Human-like arm, $\Phi=\pi / 2$, histogram and scatter plot.

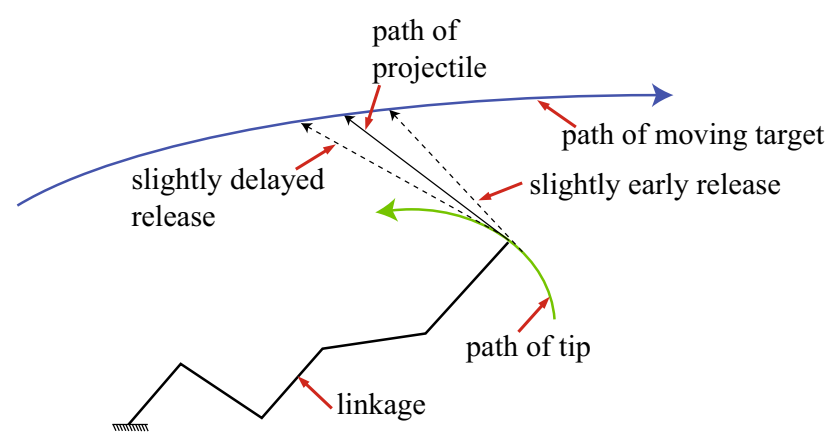

Figure 13. Directional sensitivity of the throw to small changes in the instant of release. The two dashed arrows define a triangular region, which may be thought of as accessible to the thrower.

extensive optimization and not following statistical trends, may reasonably be viewed as performing in the $96^{\text {th }}$ percentile of the population.
In this sense, even in a broad comparison set of random robots that draw no advantage from human-specific biomechanics, the human arm emerges as a potentially excellent thrower.

\section{Discussion and conclusions}

In closing, we discuss our work along three themes: its technical scope, its procedural contributions and its possible broader interpretation.

We begin by discussing the technical scope and limitations of our study. Our choice of straight-linked, fivejointed robots is somewhat restricted. Subsequent researchers may wish to allow further degrees of freedom, such as a ball-and-socket joint at the shoulder. Secondly, it is not clear what, within our robot population, is the fastestthrowing human-like arm. This limitation works in our 


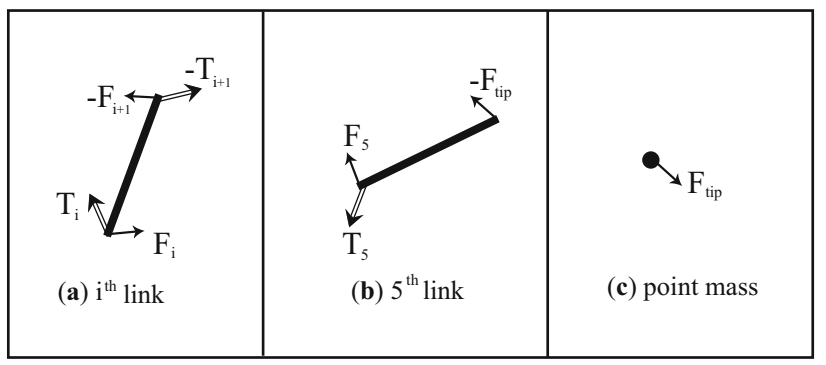

Figure 14. Free-body diagrams of the robot links.

favour, however; if there is a faster human-like throwing arm, then our point becomes stronger. Third, as admitted earlier, the optimization problem we have solved $(40,000$ times) is non-convex. We see no way to remove this difficulty; it may be alleviated only by more determined searches, greater randomization and more powerful computers. Fourth, although we find that the human-like arm is a superior thrower, our attempts to predict throwing performance in terms of robot parameters found the key predictor to be $L_{5}-M_{5}$. We do not know what other, as yet undetected, superior throwers still lie concealed within our population.

Procedurally, we have made two contributions. First, we have offered a new paradigm for viewing a variety of biomechanical tasks, of which throwing is just one. Specifically, if humans throw well, we have suggested that a useful comparison set could be mechanistic and large, as opposed to biomechanical and limited to hominids. Such a comparison may yield insights into both human throwing as well as throwing in general. Second, we have offered a practical way of seeking a large number of such optima, through our partitioning and use of the TSP solution. Future investigators may find this idea, which we have not seen elsewhere, useful.

Finally, we turn to possible broader interpretations of our work.

To begin with, we have offered fairly convincing evidence that the human-like arm seems to be a superior thrower even in a simplified mechanistic setting. Such an insight has not been offered anywhere in the literature so far. There seem to be two interesting ways to interpret this finding.

One is that even a crude approximation of a human arm throws very well relative to a large mechanistic robot population whose arms are not subject to the dimensional restrictions of the human arm. This suggests that humans are probably good throwers even somewhat outside a strictly hominid context. More interestingly, it appears that a fast-throwing human-like arm is not mechanically hard to design.

In other words, humans do indeed throw fast, and fast throwing is not inherently difficult from a mechanical viewpoint. Perhaps, in hominid evolution, the required simultaneous neurological development delayed the emergence of fast throwing.

\section{Acknowledgements}

We thank Sourav Rakshit, Taher Saif, Anurag Gupta, Devlina Chatterjee, Jim Papadopoulos, Sovan Das, Dagmar Sternad and Joe Cusumano for discussion and comments.

\section{A Robot kinematics and dynamics}

Here, we describe the kinematics and dynamics calculations for the throwing motion.

\section{A.1 Kinematics}

We use rotation matrices to calculate the positions of the links during throwing. These calculations are described here.

Consider a vector $\vec{v}$, rotated about a unit vector $\hat{n}$ by an angle $\theta$, transformed to vector $\vec{v}_{1}$. Then

$$
\vec{v}_{1}=(1-\cos \theta)(\vec{v} \cdot \hat{n}) \hat{n}+\cos \theta \vec{v}+\sin \theta(\hat{n} \times \vec{v}) .
$$

If all vectors are written as $3 \times 1$ column matrices, then Eq. (4) can be written as 8

$$
v_{1}=\left[(1-\cos \theta) n n^{T}+\cos \theta \mathbf{I}_{3}+\sin \theta \mathbf{S}_{n}\right] v
$$

where $\mathbf{I}_{3}$ is the $3 \times 3$ identity matrix and $\mathbf{S}_{n}$ is a $3 \times 3$ skewsymmetric matrix representing a cross product with $\hat{n}$. The term inside the square brackets in Eq. (5) is a $3 \times 3$ matrix, which depends on $n$ and $\theta$. We denote it by $\mathbf{R}(n, \theta)$, and call it the rotation matrix. Eq. (5) becomes

$$
v_{1}=\mathbf{R}(n, \theta) v .
$$

Now, see figure 1 . Let $\xi_{i}$ be the joint rotation about the $i^{\text {th }}$ axis $\left(n_{i}\right)$. Then

$$
\begin{gathered}
\mathbf{R}_{1}=\mathbf{R}\left(n_{1}, \xi_{1}\right), \\
\mathbf{R}_{2}=\mathbf{R}\left(\mathbf{R}_{1} n_{2}, \xi_{2}\right) \mathbf{R}_{1},
\end{gathered}
$$

and in general

$$
\mathbf{R}_{i}=\mathbf{R}\left(\mathbf{R}_{i-1} n_{i}, \xi_{i}\right) \mathbf{R}_{i-1}, \quad i \geq 2
$$

where $\mathbf{R}_{i}$ denotes the final rotation matrix for the $i^{\text {th }}$ link.

\footnotetext{
${ }^{8}$ Bold letters indicate $3 \times 3$ matrices. The $3 \times 1$ matrix counterparts of
} vectors are without the upper arrow, e.g., $\vec{v} \Leftrightarrow v$. 


\section{A.2 Dynamics}

We have used inverse dynamics, i.e., for every link, we calculate the joint torques and forces from given time histories of joint rotations. The torque calculations at any instant of interest are described here for completeness.

A.2.1 Velocities and accelerations Let $\xi_{i}$ be the $i^{\text {th }}$ joint angle rotation, $\dot{\xi}_{i}$ be its rate of change with time, and $\ddot{\xi}_{i}$ be its second time derivative. For the $i^{\text {th }}$ link, the angular velocity $\left(\vec{\omega}_{i}\right)$ is

$$
\vec{\omega}_{i}=\vec{\omega}_{i-1}+\dot{\xi}_{i} \hat{n}_{i}, \quad i \geq 1,
$$

with $\vec{\omega}_{0}$ understood to be zero; the angular acceleration $\left(\vec{\alpha}_{i}\right)$ is

$$
\vec{\alpha}_{i}=\vec{\alpha}_{i-1}+\ddot{\xi}_{i} \hat{n}_{i}+\vec{\omega}_{i-1} \times \dot{\xi}_{i} \hat{n}_{i}, \quad i \geq 1,
$$

with $\vec{\alpha}_{0}$ understood to be zero. Here, each $\hat{n}$ is rotated from its reference position to its instantaneous position using a suitable rotation matrix as given earlier.

Now, for the $i^{\text {th }}$ link, let $\vec{r}_{g, i}$ be the position vector of the centre of mass and $\vec{r}_{e, i}$ be the position vector of the end, both measured from the base of the link. The velocities of the link endpoints and centres are

$$
\vec{v}_{e, i}=\vec{v}_{e, i-1}+\vec{\omega}_{i} \times \vec{r}_{e, i}, \quad i \geq 1,
$$

and

$$
\vec{v}_{g, i}=\vec{v}_{e, i-1}+\vec{\omega}_{i} \times \vec{r}_{g, i}, \quad i \geq 1,
$$

with $\vec{v}_{e, 0}$ understood to be zero. The corresponding accelerations are

$$
\vec{a}_{e, i}=\vec{a}_{e, i-1}+\vec{\alpha}_{i} \times \vec{r}_{e, i}+\vec{\omega}_{i} \times \vec{\omega}_{i} \times \vec{r}_{e, i}, \quad i \geq 1,
$$

and

$$
\vec{a}_{g, i}=\vec{a}_{e, i-1}+\vec{\alpha}_{i} \times \vec{r}_{g, i}+\vec{\omega}_{i} \times \vec{\omega}_{i} \times \vec{r}_{g, i}, \quad i \geq 1,
$$

with $\vec{a}_{e, 0}$ understood to be zero.

A.2.2 Equations of motion Figure 14 shows free-body diagrams. $\vec{F}_{i}$ and $\vec{T}_{i}$ are forces and torques as shown. $\vec{F}_{\text {tip }}$ is the force on the point mass.

Linear momentum balance (LMB) for the point mass is

$$
\vec{F}_{\text {tip }}=m_{\text {tip }} \vec{a}_{e, 5} .
$$

LMB for the last $\left(5^{\text {th }}\right)$ link is

$$
\vec{F}_{5}-\vec{F}_{\text {tip }}=m_{5} \vec{a}_{g, 5},
$$

and for the remaining links it is

$$
\vec{F}_{i}-\vec{F}_{i+1}=m_{i} \vec{a}_{g, i}, \quad i \leq 4 .
$$

Angular momentum balance (AMB) for the fifth link is

$$
\begin{aligned}
\vec{T}_{5} & +\left(-\vec{r}_{g, 5}\right) \times \vec{F}_{5}+\left(\vec{r}_{e, 5}-\vec{r}_{g, 5}\right) \times\left(-\vec{F}_{\text {tip }}\right) \\
& =\mathbf{I}_{5}^{\mathrm{cm}} \cdot \vec{\alpha}_{5}+\vec{\omega}_{5} \times \mathbf{I}_{5}^{\mathrm{cm}} \cdot \vec{\omega}_{5},
\end{aligned}
$$

and for the remaining links it is

$$
\begin{aligned}
\vec{T}_{i} & -\vec{T}_{i+1}+\left(-\vec{r}_{g, i}\right) \times \vec{F}_{i}+\left(\vec{r}_{e, i}-\vec{r}_{g, i}\right) \times\left(-\vec{F}_{i+1}\right) \\
& =\mathbf{I}_{i}^{\mathrm{cm}} \cdot \vec{\alpha}_{i}+\vec{\omega}_{i} \times \mathbf{I}_{i}^{\mathrm{cm}} \cdot \vec{\omega}_{i}, \quad i \leq 4 .
\end{aligned}
$$

where $\mathbf{I}_{i}^{\mathrm{cm}}$ is the central moment of inertia tensor of the $i^{\text {th }}$ link.

\section{References}

[1] Roach N T, Venkadesan M, Rainbow M J and Lieberman D E 2013 Elastic energy storage in the shoulder and the evolution of high-speed throwing in Homo. Nature 498(7455): 483-486

[2] Young R W 2003 Evolution of the human hand: the role of throwing and clubbing. J. Anat. 203(1): 165-174

[3] Wood J N, Glynn D D and Hauser M D 2007 The uniquely human capacity to throw evolved from a non-throwing primate: an evolutionary dissociation between action and perception. Biol. Lett. 3: 360-364

[4] Hopkins W D, Russell J L and Schaeffer J A 2011 The neural and cognitive correlates of aimed throwing in chimpanzees: a magnetic resonance image and behavioural study on a unique form of social tool use. Philos. Trans. R. Soc. B 367: 37-47

[5] Fleisig G S, Barrentine S W, Escamilla R F and Andrews J R 1996 Biomechanics of overhand throwing with implications for injuries. Sports Med. 21(6): 421-437

[6] Cain E L, Dugas J R, Wolf R S and Andrews J R 2003 Elbow injuries in throwing athletes: a current concepts review. Am. J. Sports Med. 31(4): 621-635

[7] Braun S, Kokmeyer D and Millett P J 2009 Shoulder injuries in the throwing athlete. J. Bone Joint Surg. 91(4): 966-978

[8] Lombai F and Szederkényi G 2009 Throwing motion generation using nonlinear optimization on a 6-degree-of-freedom robot manipulator. In: Proceedings of the IEEE International Conference on Mechatronics, pp. 1-6

[9] Kato N, Matsuda K and Nakamura T 1996 Adaptive control for a throwing motion of a 2-dof robot. In: Proceedings of 4th International Workshop on Advanced Motion Control, vol. 1, pp. 203-207

[10] Sato A, Sato O, Takahashi N and Kono M 2007 Trajectory for saving energy of a direct-drive manipulator in throwing motion. Artif. Life Robot. 11(1): 61-66

[11] Senoo T, Namiki A and Ishikawa M 2008 High-speed throwing motion based on kinetic chain approach. In: IEEE/ RSJ Proceedings of International Conference on Intelligent Robots and Systems, pp. 3206-3211

[12] Kim J H, Xiang Y, Yang J, Arora J S and Abdel-Malek K 2010 Dynamic motion planning of overarm throw for a biped human multibody system. Multibody Syst. Dyn. 24(1): 1-24 
[13] Hussain I and Bari M A 2011 Mechanical analysis of overhead throwing in cricket. Int. J. Sports Sci. Eng. 5(3): 163-168

[14] Fleisig G 2001 The biomechanics of throwing. In: Proceedings of the International Symposium on Biomechanics in Sports, vol. 1(1), pp. 91-94

[15] Berkson E, Aylward R, Zachazewski J, Paradiso J and Gill T J 2006 IMU arrays: the biomechanics of baseball pitching. Orthop. J. Harv. Med. School 8: 90-94
[16] Escamilla R F, Fleisig G S, Barrentine S W, Zheng N and Andrews J R 1998 Kinematic comparisons of throwing different types of baseball pitches. J. Appl. Biomech. 14: 1-23

[17] Kernighan B W and Lin S 1970 An efficient heuristic procedure for partitioning graphs. Bell Syst. Tech. J. 49(2): 291-307

[18] Dawes R M 1979 The robust beauty of improper linear models in decision making. Am. Psychol. 34(7): 571-582

[19] Plagenhoef S, Evans F G and Abdelnour T 1983 Anatomical data for analyzing human motion. Res. Q. Exerc. Sport 54(2): 169-178 